\title{
Spectral Analysis for Evaluation of Myocardial Tracers for Medical Imaging ${ }^{1}$
}

\author{
RH Huesman, ${ }^{\dagger}$ Senior Member, IEEE; BW Reutter, ${ }^{\dagger}$ Member, IEEE; and RC Marshall ${ }^{\dagger \ddagger}$ \\ ${ }^{\dagger}$ Center for Functional Imaging, Lawrence Berkeley National Laboratory \\ University of California, Berkeley, CA 94720 USA \\ ${ }^{\ddagger}$ Martinez Veterans Affairs, Northern California Health Care System \\ Martinez, CA 94553 USA
}

\begin{abstract}
Kinetic analysis of dynamic tracer data is performed with the goal of evaluating myocardial radiotracers for cardiac nuclear medicine imaging. Data from experiments utilizing the isolated rabbit heart model are acquired by sampling the venous blood after introduction of a tracer of interest and a reference tracer. We have taken the approach that the kinetics are properly characterized by an impulse response function which describes the difference between the reference molecule (which does not leave the vasculature) and the molecule of interest which is transported across the capillary boundary and is made available to the cell. Using this formalism we can model the appearance of the tracer of interest in the venous output of the heart as a convolution of the appearance of the reference tracer with the impulse response. In this work we parameterize the impulse response function as the sum of a large number of exponential functions whose predetermined decay constants form a spectrum, and each is required only to have a nonnegative coefficient. This approach, called spectral analysis, has the advantage that it allows conventional compartmental analysis without prior knowledge of the number of compartments which the physiology may require or which the data will support.
\end{abstract}

\section{INTRODUCTION}

Kinetic analysis of dynamic tracer data is performed with the aim of quantitatively describing the uptake and washout of new and established radiopharmaceuticals with the ultimate goal of evaluating myocardial radiotracers for cardiac nuclear medicine imaging. Data from experiments utilizing the isolated rabbit heart model are acquired by sampling the venous blood after introduction of constant proportions of a tracer of interest and a reference tracer via the aorta in a retrograde manner (arterial blood). While the concentrations may change with time, the proportion of these two (or more) tracers remains constant in the arterial blood throughout each experiment. The differences in the concentrations of these tracers measured in the venous blood are the basis of the analysis and are used to estimate the clinical utility of radiotracers of biological interest.

\section{The IMPUlse Response FunCtion}

In analyzing data derived from this model, we have taken the approach that the uptake and washout of a tracer are

\footnotetext{
${ }^{1}$ This work was supported by US Department of Health and Human Services grant P01-HL25840 and by US Department of Energy contract DE-AC03-76SF00098.
}

properly characterized by an impulse response function which describes the difference between a reference molecule (which does not leave the vasculature) and the molecule of interest which is hypothesized to be transported across the capillary boundary and is made available to the cell. We use a linear systems approach in which we assume there is no interaction between molecules of the tracer or reference nor does the uptake of any molecule influence the uptake of any other. Using this formalism we can model the appearance of the tracer of interest in the venous output of the heart as a convolution of the appearance of the reference tracer with the impulse response

$$
h(t)=a h_{\mathrm{R}}(t) * g(t)=a \int_{0}^{t} h_{\mathrm{R}}(\tau) g(t-\tau) d \tau
$$

where $h(t)$ is the venous concentration of the tracer of interest as a function of time, $h_{\mathrm{R}}(t)$ is the venous concentration of the reference tracer, $a$ is the constant of proportionality of the tracer of interest relative to the reference tracer in the arterial blood, and $g(t)$ is the impulse response function. In what follows "tracer" will denote "tracer of interest" unless we specifically write "reference tracer".

We have analyzed the isolated rabbit heart data in several different ways, but each of them depends on the linearity properties illustrated in the convolution of equation (1). Differences in these analytical approaches arise from the assumptions made about the form of the impulse response function, $g(t)$. Desirable properties of $g(t)$ which can be deduced from the case where the reference tracer dispersion in the vascular blood is very small are that it be nonnegative (there should never be a negative amount of tracer in the venous blood), that it be zero for negative time (tracer should not appear in the venous blood before the reference tracer), and that its integral be less than or equal to one (the total amount of tracer which emerges in the vascular blood should not be greater than the normalized total amount of reference tracer).

In all cases several simple quantities can be used to describe the gross characteristics of the impulse response function. The overall integral of the impulse response function characterizes the fraction of molecules of interest which enter the heart and later emerge in the vascular blood. The remaining molecules enter the heart but do not reappear. These can be referred to as the bound fraction, $f_{\mathrm{B}}$.

$$
f_{\mathrm{B}}=1-\int_{0}^{\infty} g(t) d t
$$


Another quantity which can be obtained by characterization of the impulse response function is the fraction of the molecules of interest which enter the heart and emerge in the vascular blood with the same time course as molecules of the reference tracer, $f_{\mathrm{R}}$. This leads to a formulation of the impulse response as

$$
g(t)=f_{\mathrm{R}} \delta(t)+g^{*}(t)
$$

where $\delta(t)$ is the Dirac delta function. The fraction $f_{\mathrm{R}}$ are molecules which have not left the vasculature (have not been extracted from the blood). It is therefore natural to characterize that fraction of molecules which do not emerge in the vascular blood with the same time course as molecules of the reference tracer as the extraction fraction or simply as extraction, $E$.

$$
E=1-f_{\mathrm{R}}
$$

Finally there is the fraction $1-f_{\mathrm{B}}-f_{\mathrm{R}}=E-f_{\mathrm{B}}$ which is extracted by the heart and which later reappears in the venous blood and is represented in equation (3) by $g^{*}(t)$. This fraction is further characterized by the time it takes to wash out of the heart.

The impulse response function we describe here is related to the more traditional residue function as follows. Since the residue function is defined as the concentration of tracer in the myocardium, the impulse response function denoted in this work as $g^{*}(t)$ (for the venous response of extracted tracer) is proportional to the negative derivative of the impulse response for the residue function.

$$
\begin{gathered}
g_{\mathrm{RES}}(t)=E-\int_{0}^{t} g^{*}(\tau) d \tau \\
\frac{d g_{\mathrm{RES}}(t)}{d t}=-g^{*}(t)
\end{gathered}
$$

\section{PARAMETERIZATION OF THE IMPULSE RESPONSE}

We have previously used three methods to parameterize the venous impulse response of the extracted tracer, $g^{*}(t)$. In order of increasing restrictiveness they are:

1. $g^{*}(t)$ is required only to be a nonnegative, nonincreasing function $[1,2]$.

2. $g^{*}(t)$ is modeled as the sum of a large number of exponential functions whose predetermined decay constants form a spectrum, and each is required only to have a nonnegative coefficient $[3,4]$.

3. $g^{*}(t)$ is modeled as the sum of a small number of exponential functions whose decay constants and coefficients are variable $[5,6]$.

The third of these leads to the traditional method of compartmental model analysis used in nuclear medicine. The second is the subject of this work. It allows conventional compartmental analysis without prior knowledge of the number of compartments which the physiology may require or which the data will support.

The impulse response function for the spectral analysis method is given by

$$
g^{*}(t)=\sum_{j=1}^{n} \frac{c_{j}}{t_{j}} e^{-t / t_{j}}
$$

where $n$ is the number of components and $c_{j}$ is the fraction of the injected activity that has mean transit time of $t_{j}$ due to bidirectional diffusion between vasculature and cell.

\section{EXAMPLE OF SPECTRAL ANALYSIS}

Examples of spectral analysis applied to the evaluation of two myocardial flow imaging agents in a bolus injection experiment are shown in Figure 1. The spectrum of positive amplitudes for 100 exponentially decaying components are plotted for each. Time constants are equally spaced between 1 second and 190 minutes on a logarithmic scale. It is clear that ${ }^{125} \mathrm{I}$-labeled rotenone is extracted better and is retained longer than ${ }^{99 m}$ Tc-labeled sestamibi. For both, the point on the left of 1 second represents $f_{\mathrm{R}}$, the fraction which is not extracted ( 0.17 and 0.63 for rotenone and sestamibi, respectively). Figure 2 shows the spectral model fits to the time-activity curve samples for the bolus injection experiment.
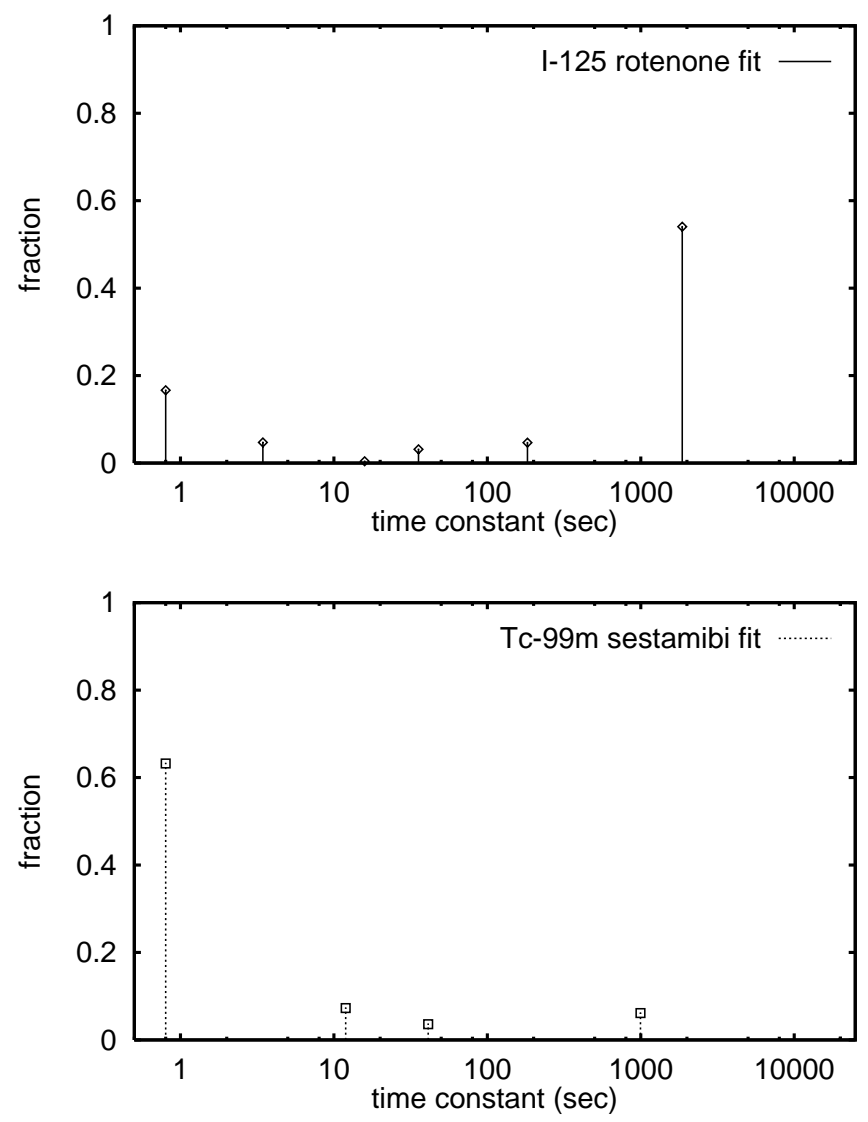

Figure 1: Spectra of exponentially decaying components. (Upper) ${ }^{125}$ I-labeled rotenone. (Lower) ${ }^{99 m}$ Tc-labeled sestamibi. 

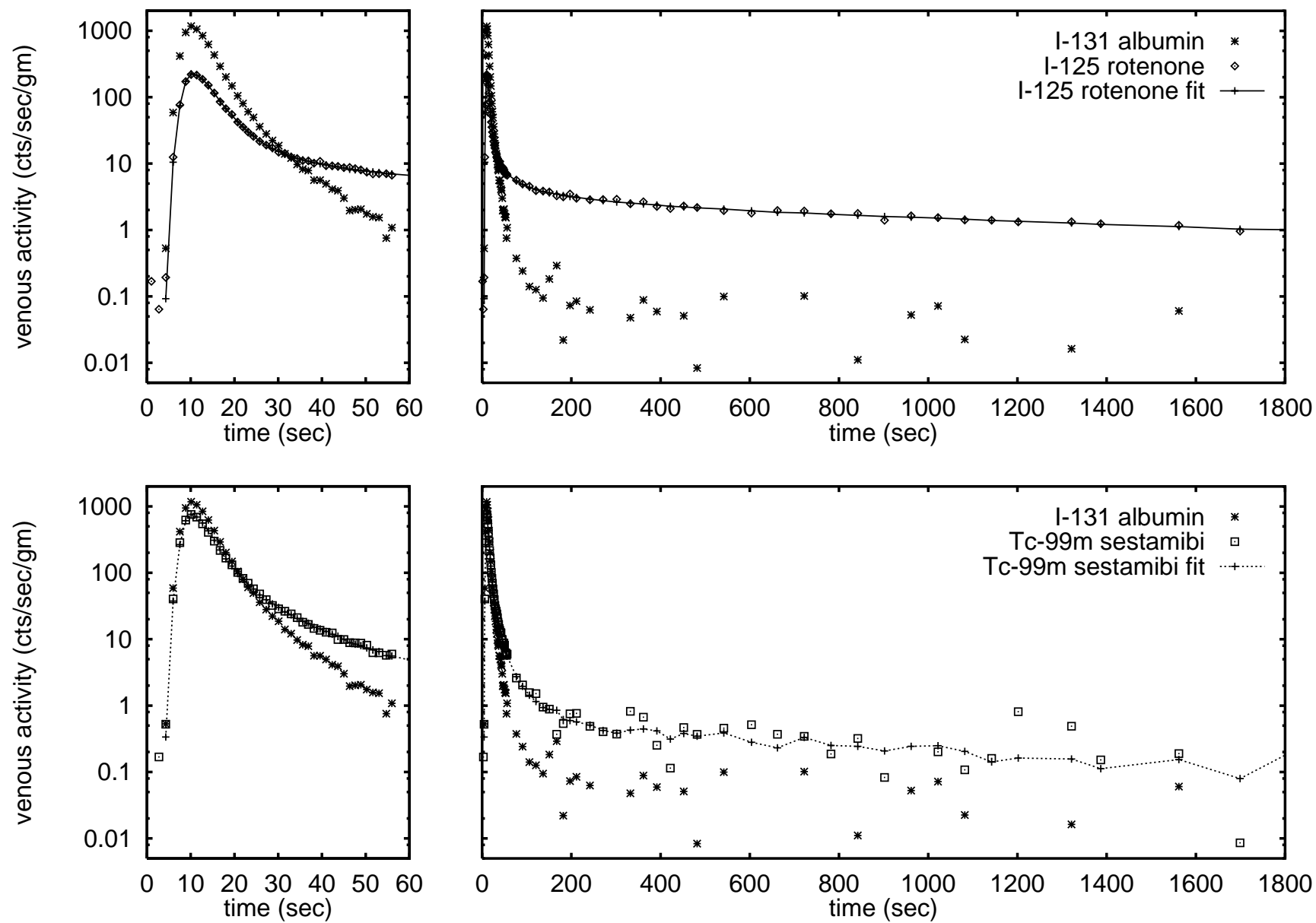

Figure 2: Time-activity curve samples and spectral model fits for a bolus injection experiment. (Upper left) ${ }^{125}$ I-labeled rotenone, first 60 sec. (Upper right) ${ }^{125}$ I-labeled rotenone, entire 30 min experiment. (Lower left) ${ }^{99 m}$ Tc-labeled sestamibi, first 60 sec. (Lower right) ${ }^{99 m}$ Tc-labeled sestamibi, entire 30 min experiment. The appearance of each myocardial flow imaging agent in the venous output of the heart was modeled as a convolution of the appearance of the reference tracer, ${ }^{131}$ I-labeled albumin, with the impulse response given by the spectral model for the flow tracer (Figure 1).

\section{ACKNOWLEDGMENT}

This work was supported by the National Heart, Lung, and Blood Institute of the US Department of Health and Human Services under grant P01-HL25840; and by the Director, Office of Science, Office of Biological and Environmental Research, Medical Sciences Division of the US Department of Energy under contract DE-AC03-76SF00098.

\section{REFERENCES}

[1] A Kuruc, W J H Caldicott, and S Treves, "An improved deconvolution technique for the calculation of renal retention functions," Comput Biomed Res, vol. 15, pp. 46-56, 1982.

[2] R C Marshall, S E Taylor, P Powers-Risius, B W Reutter, A Kuruc, P G Coxson, R H Huesman, and T F Budinger, "Kinetic analysis of rubidium and thallium as deposited myocardial blood flow tracers in isolated rabbit heart," Am J Physiol, vol. 272 (Heart Circ Physiol 41), pp. H1480H1490, 1997.
[3] V J Cunningham and T Jones, "Spectral analysis of dynamic PET studies," J Cereb Blood Flow Metab, vol. 13, no. 1, pp. 15-23, 1993.

[4] R C Marshall, P Powers-Risius, B W Reutter, S E Taylor, H F VanBrocklin, R H Huesman, and T F Budinger, "Kinetic analysis of ${ }^{125}$ I-iodorotenone as a deposited myocardial flow tracer: Comparison to ${ }^{99 m}$ Tc-sestamibi," J Nucl Med, vol. 41, 2000, (in press).

[5] R H Huesman, B L Knittel, B M Mazoyer, P G Coxson, E M Salmeron, G J Klein, B W Reutter, and T F Budinger, "Notes on RFIT: A program for fitting compartmental models to region-of-interest dynamic emission tomographic data," Report LBL-37621, Lawrence Berkeley Laboratory, 1993.

[6] R C Marshall, P Powers-Risius, R H Huesman, B W Reutter, S E Taylor, H E Maurer, M K Huesman, and T F Budinger, "Estimating glucose metabolism using glucose analogues and two tracer kinetic models in isolated rabbit heart," Am J Physiol, vol. 275 (Heart Circ Physiol 44), pp. H668-H679, 1998. 


\section{DISCLAIMER}

This document was prepared as an account of work sponsored by the United States Government. While this document is believed to contain correct information, neither the United States Government nor any agency thereof, nor The Regents of the University of California, nor any of their employees, makes any warranty, express or implied, or assumes any legal responsibility for the accuracy, completeness, or usefulness of any information, apparatus, product, or process disclosed, or represents that its use would not infringe privately owned rights. Reference herein to any specific commercial product, process, or service by its trade name, trademark, manufacturer, or otherwise, does not necessarily constitute or imply its endorsement, recommendation, or favoring by the United States Government or any agency thereof, or The Regents of the University of California. The views and opinions of authors expressed herein do not necessarily state or reflect those of the United States Government or any agency thereof, or The Regents of the University of California.

Ernest Orlando Lawrence Berkeley National Laboratory is an equal opportunity employer. 